\title{
(2) OPEN ACCESS \\ Prevalence of mismatch repair deficiency and Lynch syndrome in a cohort of unselected small bowel adenocarcinomas
}

\author{
Manon Suerink 이 , 'Gül Kilinç, ${ }^{1}$ Diantha Terlouw, ${ }^{1,2}$ Hristina Hristova, ${ }^{1}$ Lily Sensuk, \\ Demi van Egmond, ${ }^{2}$ Arantza Farina Sarasqueta, ${ }^{3}$ Alexandra M J Langers, ${ }^{4}$ \\ Tom van Wezel, ${ }^{2}$ Hans Morreau, ${ }^{2}$ Maartje Nielsen, ${ }^{1}$ PALGA-group collaborators
}

\begin{abstract}
- Additional material is published online only. To view please visit the journal online (http://dx.doi.org/10.1136/ jclinpath-2020-207040).

${ }^{1}$ Clinical Genetics, LUMC, Leiden, The Netherlands ${ }^{2}$ Pathology, LUMC, Leiden, The Netherlands ${ }^{3}$ Pathology, Amsterdam UMC Location AMC, Amsterdam, North Holland, The Netherlands ${ }^{4}$ Gastroenterology and Hepatology, LUMC, Leiden, The Netherlands

Correspondence to Manon Suerink, Clinical Genetics, LUMC, Leiden 2333ZA, The Netherlands; $m$. suerink@lumc.nl
\end{abstract}

Received 19 August 2020 Revised 25 August 2020 Accepted 26 August 2020

\begin{abstract}
Aims Previous estimates of the prevalence of mismatch repair (MMR) deficiency and Lynch syndrome in small bowel cancer have varied widely. The aim of this study was to establish the prevalence of MMR deficiency and Lynch syndrome in a large group of small bowel adenocarcinomas.

Methods To this end, a total of 400 small bowel adenocarcinomas (332 resections, 68 biopsies) were collected through the Dutch nationwide registry of histopathology and cytopathology (PathologischAnatomisch Landelijk Geautomatiseerd Archief (PALGA)). No preselection criteria, such as family history, were applied, thus avoiding (ascertainment) bias. MMR deficiency status was determined by immunohistochemical staining of MMR proteins, supplemented by MLH1 promoter hypermethylation analysis and next generation sequencing of the MMR genes.

Results MMR deficiency was observed in $22.3 \%$ of resected and $4.4 \%$ of biopsied small bowel carcinomas. Prevalence of Lynch syndrome was $6.2 \%$ in resections and $0.0 \%$ in biopsy samples. Patients with Lynch syndrome-associated small bowel cancer were significantly younger at the time of diagnosis than patients with MMR-proficient and sporadic MMRdeficient cancers (mean age of 54.6 years vs 66.6 years and 68.8 years, respectively, $p<0.000$ ).

Conclusions The prevalence of MMR deficiency and Lynch syndrome in resected small bowel adenocarcinomas is at least comparable to prevalence in colorectal cancers, a finding relevant both for treatment (immunotherapy) and family management. We recommend that all small bowel adenocarcinomas should be screened for MMR deficiency.
\end{abstract}

\section{INTRODUCTION}

Small bowel cancer is a rare form of cancer, with (c) Author(s) (or their employer(s)) 2020. Re-use permitted under CC BY-NC. No commercial re-use. See rights and permissions. Published by BMJ.

\section{To cite: Suerink $M$,} Kilinç G, Terlouw D, et al. $J$ Clin Pathol Epub ahead of print: [please include Day Month Year]. doi:10.1136/ jclinpath-2020-207040 an incidence of less than 1.0/100000, ${ }^{1}$ and little is known about the risk factors for development of this rare disease. However, monogenic cancer predisposition syndromes, such as familial adenomatous polyposis (FAP) and Lynch syndrome, are known to be responsible for a proportion of small bowel adenocarcinomas. ${ }^{2}$ While FAP, which is caused by a germline pathogenic variant in the APC gene, is characterised by the presence of polyposis coli, Lynch syndrome may be harder to recognise. ${ }^{34}$
Lynch syndrome is caused by germline pathogenic variants in one of four mismatch repair (MMR) genes (MLH1, MSH2 (EPCAM), MSH6 and $P M S 2$ ) and predisposes carriers to the development of mainly colorectal and endometrial cancer. ${ }^{4}$ In addition, risk for several other malignancies is increased, including risk for small bowel adenocarcinomas, currently estimated to be between $0.4 \%$ and $12 \%$ for $\mathrm{MLH} 1$ and $\mathrm{MSH} 2$ variant carriers. ${ }^{5}$ Unlike FAP, there are no overt clinical characteristics that distinguish a small bowel malignancy in a patient with Lynch syndrome from a sporadic case, although a personal or family history of a Lynch syndrome-associated cancer may be suggestive. Surveillance of the duodenum is generally not recommended in Lynch syndrome due to lack of evidence supporting its effectiveness. ${ }^{6}$ Nonetheless, identification of a Lynch syndrome family via a small bowel cancer case may provide the patient and other family members with the opportunity for surveillance of the colon, which has proven value as a screening strategy. ${ }^{78}$

A hallmark of Lynch syndrome-related tumours is the presence of MMR deficiency, which results from biallelic inactivation of one of the MMR genes and can be demonstrated by immunohistochemical staining of tumour tissue for the MMR proteins and/or microsatellite instability (MSI) analysis. ${ }^{9}{ }^{10}$ Lack of nuclear staining of neoplastic cells or presence of MSI is indicative of MMR deficiency. MMR deficiency in Lynch syndrome occurs due to a second somatic hit in neoplastic cells, in addition to a germline variant. MMR deficiency may also occur in sporadic cases due to somatic inactivation of both alleles. ${ }^{11}$ The presence of MMR deficiency might also be relevant to patient treatment, given that Programmed Death-Ligand 1 (PD-L1)-blockers produce a good response in MMR-deficient (colorectal) cancers regardless of sporadic or hereditary aetiology. ${ }^{11} 12$ Universal screening for MMR deficiency in small bowel cancers, as introduced for colorectal cancer and endometrial cancer in many countries, ${ }^{13} 14$ may therefore be warranted. The potential benefit of a comparable screening strategy can only be accurately assessed if the prevalence of MMR deficiency and Lynch syndrome in unselected small bowel cancer is first reliably estimated. Previous estimates of the prevalence of MMR deficiency were based on small cohorts and consequently 
showed wide variability $(0 \%-35 \%) .^{2} 15$ Few data are available on the prevalence of Lynch syndrome in these cohorts. In this study, a large, unbiased collection of small bowel cancers was used to reliably establish the prevalence of MMR deficiency and Lynch syndrome in this rare tumour group.

\section{METHODS}

\section{Cohort}

The nationwide network and registry of histopathology and cytopathology in the Netherlands, also known as PathologischAnatomisch Landelijk Geautomatiseerd Archief (PALGA), were consulted in 2017 in a nationwide search of tumour samples from patients with small bowel cancer. ${ }^{16}$ All excerpts labelled by the reporting pathologist as a neoplasm of the small bowel were extracted for the 5-year period, 2012-2016. The conclusions of the resulting pathology reports were then screened for:

1. All resected primary small bowel adenocarcinomas within the 5 -year time frame. This resulted in the selection of 411 eligible tumour specimens.

2. The hundred most recent samples that included a biopsy of an adenocarcinoma with a (possible) primary origin in the small bowel. This second category of samples was added to ensure inclusion of unresectable cases (some duodenal adenocarcinomas present at an advanced stage and are not resectable due to the high morbidity of surgery).

Formalin-fixed paraffin-embedded (FFPE) material representative of these adenocarcinomas was then requested. Material from 332 resection specimens and 68 biopsy samples was obtained. Due to the anonymous nature of the samples and the rules and regulations of the PALGA network, obtaining consent was not possible or required.

\section{Study procedures}

The study flow is visualised in figure 1 . On receipt, $4 \mu \mathrm{m}$ sections were taken from the FFPE blocks and subjected to H\&E staining and immunohistochemical staining of the MMR proteins. Additionally, depending on tumour size and histology, $10 \mu \mathrm{m}$ sections or punches from the tumour were taken for later DNA isolation. Guided by a matching H\&E slide, the $10 \mu \mathrm{m}$ sections were microdissected to enrich for tumour. All samples were coded for complete anonymity according to Dutch guidelines. Anonymous basic personal data (age at diagnosis and gender) were available for each patient, in addition to historical pathology reports. No other clinical data were available.

All adenocarcinomas were initially immunohistochemically stained for PMS2 and MSH6 protein expression. ${ }^{17}$ Subsequent immunohistochemical staining for MLH1 and/or MSH2 was performed if the tumour was PMS2 deficient or MSH6 deficient. This approach is more cost-effective than using a four-antibody panel and has good sensitivity. The rationale for this approach is that functionally, MLH1 forms a heterodimer with PMS2, while MSH2 forms a heterodimer with MSH6, and mutations in MLH1 or MSH2 result in degradation of their heterodimer partners. Hence, use of PMS2 and MSH6 antibodies as a first screening step will generally identify loss of protein expression of MLH1 or MSH2. ${ }^{17}{ }^{18}$ In cases with MLH1 deficiency, MLH1 promoter hypermethylation analysis was performed. In cases with loss of expression of MLH1 in the absence of MLH1 promoter hypermethylation or in cases with MSH2, MSH6 and solitary PMS2 expression loss, the MMR genes were further analysed using next generation sequencing (NGS). If NGS identified a variant with an allele frequency of $>40 \%$, DNA from matching non-neoplastic tissue (when available) was isolated to determine whether the variant was germline or somatic in origin.

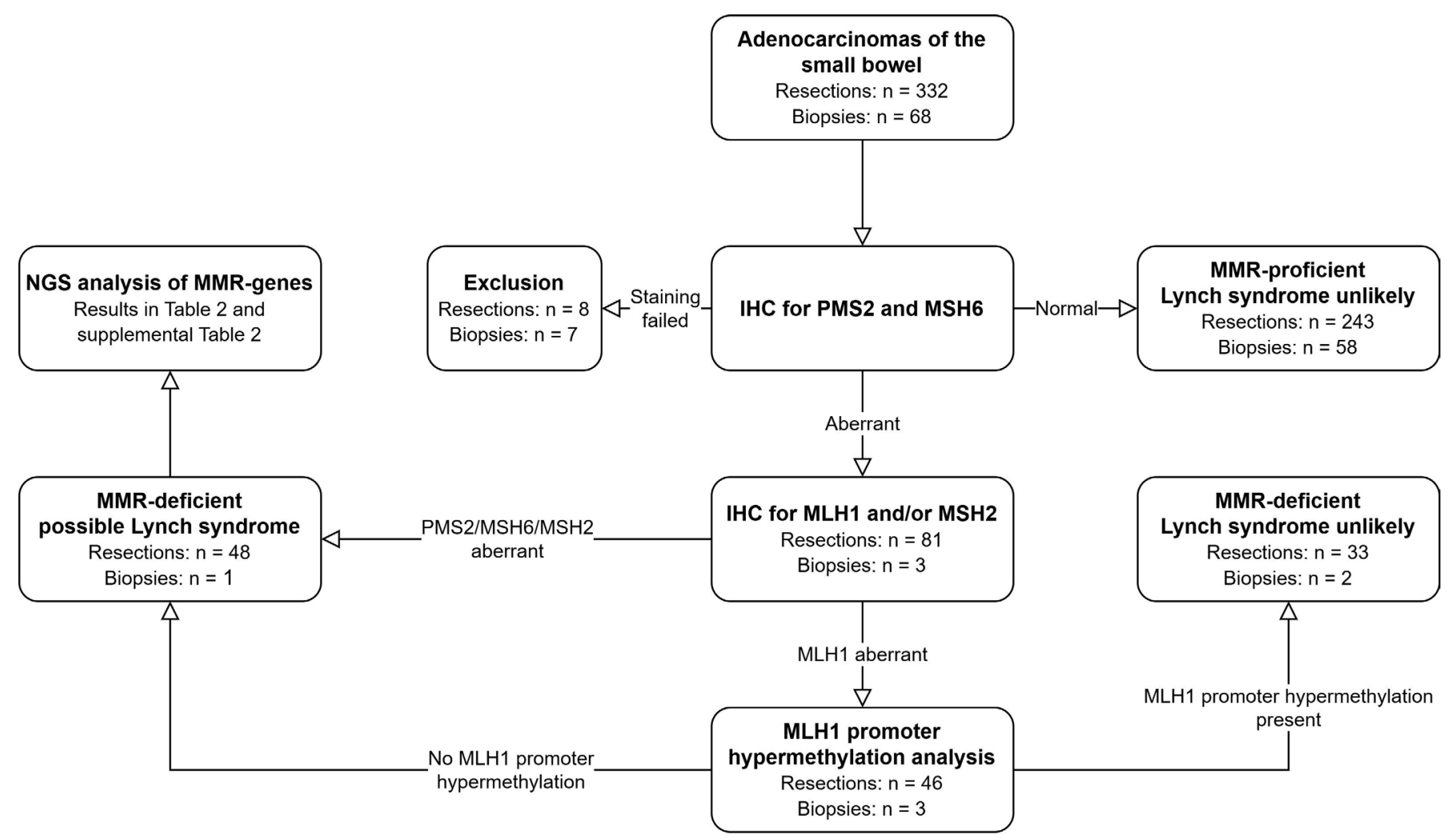

Figure 1 Study procedures. IHC, immunohistochemistry; MMR, mismatch repair; NGS, next generation sequencing. 


\section{Immunohistochemical staining}

Details on the immunohistochemical staining procedures are found in the online supplemental methods. The immunohistochemically stained samples were examined by an experienced pathologist (HM or AFS) using light microscopy to evaluate MMR status. MMR proficiency was defined as the presence of nuclear staining within neoplastic cells as well as within adjacent non-neoplastic cells. MMR deficiency was defined as an absence of nuclear staining within neoplastic cells, together with positive expression in non-neoplastic cells. A third category, subclonal loss of protein expression, was defined for those adenocarcinomas harbouring a subpopulation of cancer cells with loss of expression together with cells retaining expression of an MMR protein.

\section{DNA isolation using the tissue preparation system}

DNA was isolated using the Tissue Preparation System with VERSANT Tissue Preparation Reagents (Siemens Healthcare Diagnostics, Tarrytown, New York, USA), as previously described. ${ }^{19}$

\section{MLH1 promoter hypermethylation analysis}

Cases with loss of MLH1 expression were analysed for MLH1 promoter hypermethylation by methylation-specific PCR (MSP). ${ }^{20}$ Bisulphite conversion was carried out using the EZ DNA Methylation-Lightning Kit (D5031; Zymo Research) according to manufacturer's instructions.

\section{Targeted NGS}

Adenocarcinomas with aberrant expression of at least one of the MMR proteins in the absence of $M L H 1$ promoter hypermethylation underwent DNA variant analysis using an NGS panel. This panel consists of 20 colorectal cancer-associated and polyposisassociated genes and hotspot regions of the CTNNB1 gene (see online supplemental table 1 for all genes and panel coverage). For the purposes of this study, analysis of NGS results was restricted to MLH1, MSH2, MSH6 and PMS2. Sequencing was performed using the Ion Torrent platform according to the manufacturer's recommendations. Details are found in the online supplemental methods.

The unaligned sequence reads generated by the sequencer were mapped against a human reference genome (hg19) using the Burrows-Wheeler aligner. VarScan and ANNOVAR software were used for variant calling and annotation, respectively, and Integrative Genomics Viewer (IGV) software was used to visualise the read alignment and presence of variants. Additionally, the Leiden Open Variant Database, ClinVar and Alamut software were used whenever additional variant interpretation was needed.

\section{Statistical analysis}

Using IBM SPSS Statistics V.24, the $\chi^{2}$ test and one-way analysis of variance (ANOVA) test were performed as appropriate to compare patient and tumour characteristics of MMR-proficient cases with sporadic MMR-deficient cases and Lynch syndromeassociated cases. A p value $<0.05$ was considered to be statistically significant. Cases with subclonal loss of one of the MMR proteins were excluded from these analyses.

\section{RESULTS}

\section{Immunohistochemistry}

The prevalence of MMR deficiency, as determined by immunohistochemical staining, was $22.3 \%$ in resected small bowel
Table 1 Prevalence of mismatch repair (MMR) deficiency and immunohistochemical staining patterns in resected and biopsied adenocarcinoma samples

\begin{tabular}{lcc}
\hline Immunohistochemistry results & $\begin{array}{l}\text { Resections } \\
\text { N (\%) }\end{array}$ & $\begin{array}{l}\text { Biopsies } \\
\text { N (\%) }\end{array}$ \\
\hline MMR proficient & $243(73.2)$ & $58(85.3)$ \\
\hline MMR deficiency_complete tumour & $74(22.3)$ & $3(4.4)$ \\
\hline MLH1/PMS2 & 42 & 3 \\
\hline PMS2 only & 7 & 0 \\
\hline MSH2/MSH6 & 19 & 0 \\
\hline MSH6 only & 6 & 0 \\
Subclonal MMR deficiency & $7(2.1)$ & $0(0)$ \\
MLH1/PMS2 & 4 & \\
MSH6 only & 1 & \\
All four deficient & 2 & \\
No tumour, excluded from further analysis & $8(2.4)$ & $7(10.3)$ \\
\hline Total & 332 & 68 \\
\hline
\end{tabular}

adenocarcinomas and $4.4 \%$ in biopsies (table 1). Additionally, seven $(2.1 \%)$ resected samples showed subclonal loss of at least one MMR protein. Eight resected adenocarcinomas and seven adenocarcinoma biopsy samples had to be excluded from further analysis because no (representative) tumour tissue was present in the available FFPE blocks.

\section{Causes of mismatch repair deficiency}

The most common cause of MMR deficiency was MLH1 promoter hypermethylation (40.5\% of MMR-deficient resections and $66.7 \%$ of MMR-deficient biopsies, table 2). In more than a quarter of MMR-deficient resection samples, the MMR deficiency was related to Lynch syndrome (27\%, table 2 and online supplemental table 2). The prevalence of Lynch syndrome within the total resection cohort was therefore at least 20/324 (6.2\%). The true number might in fact be higher because in six cases an MMR gene variant with a high allele frequency $(>40 \%$ of reads) was identified within the tumour, but matched normal tissue was not available to confirm or refute germline origin of the variant.

\begin{tabular}{|c|c|c|c|}
\hline & \multicolumn{2}{|c|}{$\begin{array}{l}\text { MMR-deficient } \\
\text { tumours }\end{array}$} & \multirow{2}{*}{$\begin{array}{l}\text { Subclonal } \\
\text { loss N (\%) } \\
\text { Resections } \\
\mathrm{N}(\%)\end{array}$} \\
\hline & $\begin{array}{l}\text { Resections } \\
\mathrm{N}(\%)\end{array}$ & $\begin{array}{l}\text { Biopsies } \\
\mathrm{N}(\%)\end{array}$ & \\
\hline MLH1 promoter hypermethylation & $30(40.5)$ & $2(66.7)$ & $3(42.9)$ \\
\hline Two somatic hits & $10(13.5)$ & 0 & $1(14.3)$ \\
\hline Lynch syndrome & $20(27.0)$ & 0 & 0 \\
\hline MLH1 variant & 6 & & \\
\hline MSH2 variant & 7 & & \\
\hline PMS2 variant & 2 & & \\
\hline MSH6 variant & 5 & & \\
\hline $\begin{array}{l}\text { MMR variants identified in tumour, normal } \\
\text { tissue not available, but high variant allele } \\
\text { frequency }\end{array}$ & $6(8.1)$ & 0 & 0 \\
\hline $\begin{array}{l}\text { MMR deficiency molecularly unexplained (no or } \\
\text { only one somatic hit identified) }\end{array}$ & $8(10.8)$ & $1(33.3)$ & $3(42.9)$ \\
\hline Total & 74 & 3 & 7 \\
\hline
\end{tabular}


Table 3 Cohort characteristics for Lynch syndrome versus mismatch repair (MMR) proficient versus MMR-deficient cases

\begin{tabular}{|c|c|c|c|c|c|}
\hline & & $\begin{array}{l}\text { MMR-proficient } \\
\mathrm{N}=243\end{array}$ & $\begin{array}{l}\text { Sporadic MMR-deficient } \\
\text { carcinomas } \mathrm{N}=44\end{array}$ & $\begin{array}{l}\text { Lynch syndrome } \\
\mathrm{N}=20\end{array}$ & $P$ value \\
\hline \multicolumn{2}{|c|}{ Gender-male } & $126(51.9 \%)$ & $23(52.3 \%)$ & $13(65.0 \%)$ & 0.525 \\
\hline \multicolumn{2}{|c|}{ Mean age at diagnosis in years (range) } & $66.6(27-91)$ & $68.8(43-90)$ & $54.6(35-77)$ & $<0.000$ \\
\hline \multirow{2}{*}{ Location (\%) } & Jejunum & $51(21.0 \%)$ & $7(15.9 \%)$ & $3(15.0 \%)$ & \\
\hline & lleum & $33(13.6 \%)$ & $4(9.1 \%)$ & $3(15.0 \%)$ & \\
\hline \multicolumn{2}{|c|}{ Previous history of other cancer type(s) (non-Lynch) $\uparrow$} & $27(11.1 \%)$ & $6(13.6 \%)$ & $6(30.0 \%)$ & 0.050 \\
\hline \multicolumn{2}{|c|}{ Crohn's disease-yes } & $8(3.3 \%)$ & $0(0 \%)$ & $0(0 \%)$ & 0.339 \\
\hline \multicolumn{2}{|c|}{ Coeliac disease-yes } & & & & \\
\hline
\end{tabular}

*Lynch syndrome-associated cancers: colorectal cancer, endometrial cancer, ovarian cancer, gastric cancer, cancer of the bile duct or gallbladder, pancreatic cancer or urothelial cancer (Møller et $a^{22}$ ).

tExcluding basal cell cancer of the skin.

\section{Comparison of patient and tumour characteristics}

A comparison of patient and tumour characteristics of MMRproficient, (apparently) sporadic MMR-deficient and Lynch syndrome-associated cases included only the resected adenocarcinoma cases, as they represent the largest subcohort and have a documented primary tumour location within the small bowel. The six cases carrying a high allele frequency variant but without available-matched normal tissue were excluded due to uncertainty regarding their status as Lynch syndrome or sporadic MMR-deficient cases. Cases with an unexplained MMR deficiency and those with subclonal MMR deficiencies were also excluded from this analysis.

Mean age at cancer diagnosis was significantly lower in the patients with Lynch syndrome (table 3), and a previous history of a Lynch syndrome-associated cancer was significantly elevated in patients with Lynch syndrome. Interestingly, coeliac disease (diagnosed based on pathology reports of small bowel biopsies unconnected to the small bowel cancer diagnosis) was significantly more common in sporadic MMR-deficient cases. No other significant associations were identified (eg, location, gender, other cancer history, ${ }^{22}$ Crohn's disease).

\section{DISCUSSION}

In a large group of resected primary small bowel adenocarcinomas, we found complete MMR deficiency in $22.3 \%$ and subclonal deficiency in $2.1 \%$ of cases, while biopsied small bowel adenocarcinomas showed a lower prevalence of MMR deficiency (4.4\%). To the best of our knowledge, this is the first study to systematically screen a large, consecutive group of small bowel adenocarcinomas for the prevalence of MMR deficiency. Previous studies were either smaller and/or used selected cases with a higher a priori chance of being related to Lynch syndrome. Furthermore, many of these studies did not include molecular analysis to verify whether MMR deficiency was Lynch syndrome-related or sporadic. ${ }^{215} 23$

A recently published French study by Aparicio $e t \mathrm{al}^{24}$ reported a Lynch syndrome prevalence of $6.9 \%$ in a large cohort of small bowel adenocarcinomas, in line with a prevalence of at least $6.2 \%$ in our cohort. MMR deficiency prevalence could not be compared because this French cohort was not systematically screened for MMR deficiency.

Of particular note, the prevalence of MMR deficiency in our study differed considerably between the resected and biopsied specimens. A higher prevalence of MMR deficiency in resected versus biopsied samples might be related to the association of MMR deficiency with a better prognosis in other cancers, ${ }^{25}$ so resections may represent patients with cancer with a relatively good prognosis, whereas biopsies may represent patients with a poor prognosis who are less likely to undergo resection. Interestingly, the prevalence of MMR deficiency identified in biopsied samples, $4.4 \%$, is close to the $5.0 \%$ prevalence identified in a metastatic colorectal cancer cohort. ${ }^{26}$ However, as no further clinical data were available to verify that a biopsied sample was a confirmed primary small bowel cancer, our cohort may also have included cancers with a different primary location (where MMR deficiency prevalence is lower). Further validation of the prevalence of MMR deficiency in a cohort of small bowel cancers that were not resected is therefore required.

The relevance of subclonal loss of MMR protein expression is still poorly understood. While it seems unlikely that these patients have Lynch syndrome, the relevance of subclonal loss for prognosis and/or therapy will require further investigation. ${ }^{18} 27$

A significant overrepresentation of patients with coeliac disease was noted among cases with sporadic MMR deficiency. An association of coeliac disease with sporadic MMR deficiency (particularly with MLH1 promoter hypermethylation) has been described previously, ${ }^{28} 29$ and two out of three MMR-deficient cases from our cohort also showed MLH1 promoter hypermethylation. A limitation of our study was the lack of accompanying clinical data, which meant that we had no information on treatment/diet and could not verify whether the pathological signs of coeliac disease correlated with patient symptoms. These results should therefore be interpreted with caution, because there are other conditions that mimic the histological signs of coeliac disease. $^{30}$

Another drawback of anonymous data is that it precludes verification of the number of Lynch syndrome cases, knowledge that might otherwise be used to establish how many patients are missed using current practices. Nevertheless, from pathology reports we could deduce that 13 out of 20 patients with Lynch were likely already identified, either because MSI and/or immunohistochemical testing was described (in the small bowel tumour or a previous tumour) or a previous diagnosis of Lynch syndrome was mentioned (online supplemental table 3 ).

There is an ongoing discussion whether a two-antibody panel for immunohistochemical staining of the MMR proteins has sufficient sensitivity to detect MMR-deficient cases. Although a small number of MMR-deficient cases may be missed with a 
two-antibody panel, it is not expected that the results of a fourantibody approach would alter our conclusions.

A molecular cause of MMR deficiency could not always be identified $(n=12)$. This is likely partly explained by the fact that we did not perform multiplex ligation-dependent probe amplification analysis to screen samples for deletions and/or insertions (germline or somatic) of the MMR genes or EPCAM (table 2 and online supplemental table 2). Nonetheless, NGS data were manually checked using the IGV for evidence suggesting a deletion, which led to the identification of deletions in three samples (online supplemental table 2, eg, study ID 33). Although this approach lowers the risk of missing copy number variants, not all deletions/insertions will be identified. As EPCAM was not sequenced, deletions of this gene will have been missed by definition. However, as only 1\%-3\% of all Lynch syndrome families carry an EPCAM deletion and deletions/insertions of the MMR genes explain a minority of Lynch syndrome families, ${ }^{431}$ Multiplex Ligation-dependent Probe Amplification (MLPA) analysis is unlikely to have altered our conclusions and recommendations. Another possible explanation for the failure of NGS results to resolve all MMR deficiency cases is that some cases lacked the informative single nucleotide polymorphisms required to determine whether loss of heterozygosity has occurred.

The analysis of PMS2 is complicated by the presence of pseudogenes. Nevertheless, researchers from our group have shown that it is possible to reliably detect variants in PMS2, even when using DNA isolated from FFPE material, as long as the correct amplicons are selected. ${ }^{32}$ Exceptions include variants in exon 12-15 due to gene conversion. The two germline variants identified in our cohort are found in exons 1-11.

In our cohort, the prevalence of MMR deficiency in resected cases (22.3\%) was higher than the reported prevalence of MMR deficiency in colorectal cancer $(15 \%) .{ }^{33}$ This finding has implications for daily clinical practice in relation to three important issues: prognosis, treatment and surveillance. In (early stage) colorectal cancer, MMR deficiency has been linked to a better prognosis, 253435 an association that may also hold true for MMR-deficient small bowel cancers. Indeed, the aforementioned study by Aparicio et al reported a trend towards better prognosis for Lynch-associated small bowel adenocarcinomas versus those related to Crohn's disease. ${ }^{24}$ Furthermore, with the advent of immunoblockade therapy and its proven efficacy in MMR-deficient cancers, ${ }^{36}$ MMR status is relevant when formulating treatment strategies regardless of germline or sporadic status. Finally, due to the high prevalence of Lynch syndrome, small bowel cancer as an entity may facilitate the identification of new Lynch syndrome families and consequently allow surveillance measures to be offered.

In light of the high prevalence of MMR deficiency and Lynch syndrome, together with associated relevance and benefits, we recommend the implementation of universal screening of all

Take home messages

- Prevalence of mismatch repair deficiency (22.3\%) and Lynch syndrome (6.2\%) in resected small bowel adenocarcinomas is high.

- No clear clinical or histological predictors of mismatch repair deficiency were identified.

- We recommend the implementation of universal screening for mismatch repair deficiency in all small bowel adenocarcinomas. primary small bowel adenocarcinomas for the presence of MMR deficiency. An age limit of 70 years is often used in the universal screening of colorectal cancers for mismatch repair deficiency. However, as the Lynch syndrome-associated cases included in our study showed a very broad age range (35-77 years, table 3 ) at diagnosis, we suggest that age limits on universal screening for small bowel cancer may be detrimental.

\section{Handling editor Runjan Chetty.}

Twitter Manon Suerink @ManonSuerink and Hristina Hristova @HristinaHr

Acknowledgements We thank Medactie.com for assistance with the editing of this manuscript. We thank our PALGA-group collaborators for providing patient samples: Dr E.J.M. Ahsmann, Klinische pathologie Groene Hart Ziekenhuis; Dr C. Jansen, Laboratorium Pathologie Oost-Nederland; R.S. van der Post, Radboud UMC Nijmegen; C. Wauters, CWZ Nijmegen; Dr C.Y. Yick, Amphia Ziekenhuis Breda.

Collaborators PALGA-group collaborators: Dr E J M Ahsmann, Groene Hart Ziekenhuis; Dr C Jansen, Laboratorium Pathologie Oost-Nederland; R S van der Post, Radboud UMC Nijmegen; C Wauters, CWZ Nijmegen; Dr C Y Yick, Amphia Ziekenhuis Breda.

Contributors MN, HM and TVW were the overall principal investigators in this study, they conceived the study and were responsible for the study design and supervision of the entire study. MN obtained financial support. MS and GK performed statistical analyses, interpreted the results and drafted the initial manuscript. MS, GK, DT, DvE, LS and HH performed laboratory experiments. MS, GK, LS and HH were responsible for sample preparation. HM and AFS assessed all pathology slides (H\&E and immunohistochemical staining). AMJL assisted in the interpretation of the results, and reviewed and contributed to the writing of the manuscript. PALGA group provided patient samples. All authors approved the final report for publication.

Funding This work was supported by a grant from the Dutch Cancer Society (KWF UL 2012-5155).

Competing interests None declared.

\section{Patient consent for publication Not required.}

Ethics approval A favourable ethical opinion was received from the Medical Ethical Review Board of Leiden University Medical Centre (reference number P16.313)

Provenance and peer review Not commissioned; internally peer reviewed.

Data availability statement Data are available upon reasonable request. Our data consist of deidentified participant data and are available upon reasonable request by contacting the corresponding author (m.suerink@lumc.nl).

Open access This is an open access article distributed in accordance with the Creative Commons Attribution Non Commercial (CC BY-NC 4.0) license, which permits others to distribute, remix, adapt, build upon this work non-commercially, and license their derivative works on different terms, provided the original work is properly cited, appropriate credit is given, any changes made indicated, and the use is non-commercial. See: http://creativecommons.org/licenses/by-nc/4.0/.

\section{ORCID iD}

Manon Suerink http://orcid.org/0000-0001-7058-6973

\section{REFERENCES}

1 Pan SY, Morrison H. Epidemiology of cancer of the small intestine. World J Gastrointest Oncol 2011;3:1-42.

2 Aparicio T, Zaanan A, Mary F, et al. Small bowel adenocarcinoma. Gastroenterol Clin North Am 2016;45:447-57.

3 Jasperson KW, Patel SG, Ahnen DJ. APC-Associated Polyposis Conditions. In: Adam MP Ardinger HH, Pagon RA, eds. GeneReviews R. Seattle (WA, 1993.

4 Kohlmann W, Gruber SB. Lynch Syndrome. In: Adam MP, Ardinger HH, Pagon RA, eds. GeneReviews R. Seattle (WA, 1993.

5 Giardiello FM, Allen Jl, Axilbund JE, et al. Guidelines on genetic evaluation and management of Lynch syndrome: a consensus statement by the US Multi-Society Task force on colorectal cancer. Gastroenterology 2014;147:502-26.

6 Koornstra JJ, Kleibeuker JH, Vasen HFA. Small-Bowel cancer in Lynch syndrome: is it time for surveillance? Lancet Oncol 2008;9:901-5.

7 Vasen HFA, Abdirahman M, Brohet R, et al. One to 2-year surveillance intervals reduce risk of colorectal cancer in families with Lynch syndrome. Gastroenterology 2010;138:2300-6.

8 de Vos tot Nederveen Cappel WH, Järvinen HJ, Lynch PM, et al. Colorectal surveillance in Lynch syndrome families. Fam Cancer 2013;12:261-5. 
9 Yoon YS, Yu CS, Kim TW, et al. Mismatch repair status in sporadic colorectal cancer: immunohistochemistry and microsatellite instability analyses. J Gastroenterol Hepatol 2011;26:1733-9.

10 Cerretelli G, Ager A, Arends MJ, et al. Molecular pathology of Lynch syndrome. J Pathol 2020;250:518-31.

11 Battaglin F, Naseem M, Lenz H-J, et al. Microsatellite instability in colorectal cancer: overview of its clinical significance and novel perspectives. Clin Adv Hematol Oncol 2018;16:735-45.

12 Le DT, Durham JN, Smith KN, et al. Mismatch repair deficiency predicts response of solid tumors to PD-1 blockade. Science 2017;357:409-13.

13 Vindigni SM, Kaz AM. Universal screening of colorectal cancers for Lynch syndrome: challenges and opportunities. Dig Dis Sci 2016;61:969-76.

14 Dillon JL, Gonzalez JL, DeMars L, et al. Universal screening for Lynch syndrome in endometrial cancers: frequency of germline mutations and identification of patients with Lynch-like syndrome. Hum Pathol 2017;70:121-8.

15 Jun S-Y, Lee E-J, Kim M-J, et al. Lynch syndrome-related small intestinal adenocarcinomas. Oncotarget 2017;8:21483-500.

16 Casparie M, Tiebosch ATMG, Burger G, et al. Pathology databanking and biobanking in the Netherlands, a central role for PALGA, the nationwide histopathology and cytopathology data network and archive. Cell Oncol 2007;29:19-24.

17 Mojtahed A, Schrijver I, Ford JM, et al. A two-antibody mismatch repair protein immunohistochemistry screening approach for colorectal carcinomas, skin sebaceous tumors, and gynecologic tract carcinomas. Mod Pathol 2011:24:1004-14

18 Stelloo E, Jansen AML, Osse EM, et al. Practical guidance for mismatch repairdeficiency testing in endometrial cancer. Ann Oncol 2017;28:96-102.

19 van Eijk R, Stevens L, Morreau H, et al. Assessment of a fully automated highthroughput DNA extraction method from formalin-fixed, paraffin-embedded tissue for KRAS, and BRAF somatic mutation analysis. Exp Mol Pathol 2013;94:121-5.

20 van Roon EH, Boot A, Dihal AA, et al. Braf mutation-specific promoter methylation of Fox genes in colorectal cancer. Clin Epigenetics 2013;5:2.

21 Pérez-Carbonell L, Alenda C, Payá A, et al. Methylation analysis of MLH1 improves the selection of patients for genetic testing in Lynch syndrome. J Mol Diagn 2010;12:498-504.

22 Møller P, Seppälä TT, Bernstein I, et al. Cancer risk and survival in path_MMR carriers by gene and gender up to 75 years of age: a report from the Prospective Lynch Syndrome Database. Gut 2018;67:1306-16.
23 Latham A, Srinivasan P, Kemel Y, et al. Microsatellite instability is associated with the presence of Lynch syndrome pan-cancer. J Clin Oncol 2019;37:286-95.

24 Aparicio T, Henriques J, Manfredi S, et al. Small bowel adenocarcinoma: results from a nationwide prospective ARCAD-NADEGE cohort study of 347 patients. Int I Cancer 2020;147:967-77.

25 Deng Z, Qin Y, Wang J, et al. Prognostic and predictive role of DNA mismatch repair status in stage II-III colorectal cancer: a systematic review and meta-analysis. Clin Genet 2020:97:25-38.

26 Venderbosch S, Nagtegaal ID, Maughan TS, et al. Mismatch repair status and BRAF mutation status in metastatic colorectal cancer patients: a pooled analysis of the Cairo, CAIRO2, coin, and focus studies. Clin Cancer Res 2014;20:5322-30.

27 Chen W, Pearlman R, Hampel H, et al. Msh6 immunohistochemical heterogeneity in colorectal cancer: comparative sequencing from different tumor areas. Hum Pathol 2020;96:104-11

28 Vanoli A, Di Sabatino A, Furlan D, et al. Small bowel carcinomas in coeliac or Crohn's disease: clinico-pathological, molecular, and prognostic features. A study from the small bowel cancer Italian Consortium. J Crohns Colitis 2017:11:942-53.

29 Rizzo F, Vanoli A, Sahnane N, et al. Small-Bowel carcinomas associated with celiac disease: transcriptomic profiling shows predominance of microsatellite instabilityimmune and mesenchymal subtypes. Virchows Arch 2020;476:711-723.

30 Kamboj AK, Oxentenko AS. Clinical and histologic mimickers of celiac disease. Clin Trans/ Gastroentero/ 2017:8:e114.

31 Kuiper RP, Vissers LELM, Venkatachalam R, et al. Recurrence and variability of germline EpCAM deletions in Lynch syndrome. Hum Mutat 2011;32:407-14.

32 Jansen AML, Tops CMJ, Ruano D, et al. The complexity of screening pms 2 in DNA isolated from formalin-fixed paraffin-embedded material. Eur J Hum Genet 2020;28:333-8.

33 Vilar E, Gruber SB. Microsatellite instability in colorectal cancer-the stable evidence. Nat Rev Clin Oncol 2010;7:153-62.

34 Benson AB, Venook AP, Cederquist $L$, et al. Colon cancer, version 1.2017, NCCN clinical practice guidelines in oncology. J Nat/ Compr Canc Netw 2017;15:370-98.

35 Wang B, Li F, Zhou X, et al. Is microsatellite instability-high really a favorable prognostic factor for advanced colorectal cancer? A meta-analysis. World J Surg Oncol 2019;17:169.

36 Zhao $\mathrm{P}$, Li L, Jiang $\mathrm{X}$, et al. Mismatch repair deficiency/microsatellite instabilityhigh as a predictor for anti-PD-1/PD-L1 immunotherapy efficacy. J Hematol Oncol 2019;12:54. 